\title{
Urinary orosomucoid: validation of an automated immune turbidimetric test and its possible clinical use
}

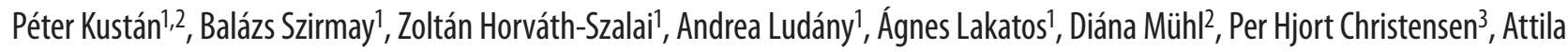 \\ Miseta', Gábor L. Kovács ${ }^{1,4}$, Tamás Köszegi*1,4 \\ 1'Department of Laboratory Medicine, University of Pécs Medical School, Pécs, Hungary \\ 2Department of Anaesthesiology and Intensive Therapy, University of Pécs Medical School, Pécs, Hungary \\ ${ }^{3}$ Dako A/S, Glostrup, Denmark \\ ${ }^{4}$ Szentágothai Research Centre, Pécs, Hungary \\ *Corresponding author: tamas.koszegi@aok.pte.hu
}

\begin{abstract}
Introduction: Besides routine serum markers of inflammatory diseases, the diagnostic potential of selected urinary proteins has not been fully exploited yet. Former studies revealed that urinary orosomucoid (u-ORM) might have complementary information in inflammatory disorders. Our aim was to develop and validate a fully automated method for U-ORM measurements and to evaluate its potential clinical impact on systemic inflammatory diseases.

Materials and methods: A particle-enhanced immune turbidimetric assay was validated for a Cobas 8000/c502 analyzer to determine u-0RM levels. Spot urine samples from 72 healthy individuals, 28 patients with Crohn's disease and 30 septic patients were studied.

Results: Our assay time was 10 minutes and the detection limit of u-ORM was $0.02 \mathrm{mg} / \mathrm{L}$. The intra- and inter-assay imprecision expressed as CV was less than $5 \%$, and the recovery ranged between $95-103 \%$. Within 10 to 60 years of age, a preliminary reference range for urinary orosomucoid/ creatinine ratio ( $\mathrm{u}-0 \mathrm{RM} / \mathrm{u}-\mathrm{CREAT}$ ) was found to be $0.08(0.01-0.24) \mathrm{mg} / \mathrm{mmol}$ [median (2.5-97.5 percentiles)]. Compared to controls, a five-fold increase of u-ORM/u-CREAT values in Crohn's disease and approximately a 240-fold increase in sepsis were observed.

Conclusions: We set up a fast, sensitive and precise turbidimetric approach for automated u-ORM determination. Our highly sensitive assay is ideal for routine $\mathrm{u}-\mathrm{ORM}$ measurements and might be a potential novel laboratory test in the management of systemic inflammatory processes.
\end{abstract}

Key words: orosomucoid; urine; particle-enhanced turbidimetry; inflammation; biomarker

\section{Introduction}

The leading causes of global mortality - cardiovascular diseases, diabetes mellitus, malignancies and infections - and their complications are all associated with activation of the inflammatory system. Laboratory parameters are fundamental in these disorders not only for early diagnosis, but also for the detection of complications. Furthermore, biomarkers are particularly useful in risk stratification, prediction of disease progression and outcome. It is additionally advantageous, if a marker can be measured from a non-invasively obtained sample by a fast, automated routine diagnostic procedure.
Human orosomucoid (a-1-acid glycoprotein, ORM) is an extensively glycosylated, abundant constituent of the a-1-globulin family (1). It is an acute phase protein produced mainly by the liver with a molecular mass of 41-43 kDa; however, lower amounts of ORM can be also synthesized by leukocytes and endothelial cells (2). As a member of the lipocalin protein family, ORM plays a role in transporting biomolecules and drugs (3). Furthermore, it has been revealed that ORM takes part in maintaining capillary perm-selectivity, representing its immunomodulatory effect $(4,5)$. 
As an acute phase protein, serum concentrations of ORM can increase up to two-three fold in malignancies, inflammatory diseases and severe infection, thus it serves as a general, non-specific inflammatory marker $(6,7)$. ORM can also be found in the urine with much lower concentrations than in serum. However, information on its role in the urine is limited. Most probably it is filtrated through the glomeruli, but the mechanism of urinary excretion of ORM is not well clarified (8).

Former studies revealed that ORM is a considerably abundant component of urinary proteins (821). Markedly increased urinary excretion of ORM was found after physical exercise, in acute inflammation, preeclampsia, lupus and also in bladder cancer (9-14). In spite of the fact that the elevation of urinary orosomucoid (u-ORM) concentrations seems to be a non-specific marker, several publications stated that u-ORM might serve advantageous information on early monitoring of certain inflammatory disorders. Elevated u-ORM was described in diabetes mellitus associated with the ongoing chronic low-grade inflammation and endothelial dysfunction and was considered to be an independent predictor of diabetic nephropathy and cardiovascular mortality among patients with type 2 diabetes (15-19). Recent findings suggest that it also might be a potential novel marker for the early detection of chronic heart failure (20). Previously we showed that u-ORM could be a more sensitive marker of inflammation than serum ORM which rises only 2 -fold in contrast to even 200-fold increase of u-ORM in sepsis (21). Others also showed that the elevation of $u$-ORM is much more considerable than that of serum ORM in relation to inflammatory diseases $(10,11,17)$. These results indicate that $\mathrm{u}$-ORM is a reliable marker of chronic and acute inflammatory disorders and provides valuable supplementary information for daily clinical practice.

In spite of promising literature data on the potential utility of u-ORM, a commercially available, sensitive, fast and fully automated laboratory test for u-ORM measurement is unavailable yet. Several different methods exist to determine u-ORM (western blotting, radioimmunoassay, ELISA), though all of these analyses are not appropriate for routine work. In our previous studies we performed an in-house western blot method and a laser nephelometric assay for u-ORM measurements in several patient groups and in healthy individuals $(21,22)$. Although we could determine ORM values in urine, neither techniques are usable in daily routine: western blotting is time consuming; moreover, it is not accurate enough, while nephelometry was not properly sensitive for values found in healthy individuals. Clinical application requires a simple, reliable, fast and sensitive automated method. For this purpose, we decided to adapt and validate a particle-enhanced turbidimetric assay on a fully automated clinical chemistry analyzer for the quantitative determination of ORM in urine. Our further aim was to determine preliminary reference ranges of u-ORM in different age groups of healthy individuals and to investigate its applicability in acute and chronic inflammatory diseases.

\section{Materials and methods}

\section{Methods}

\section{Instrumentation, reagents, assay conditions}

The latex particle-enhanced turbidimetric (PET) assay for u-ORM measurement was carried out on the $c 502$ module of a Cobas 8000 analyzer (Roche Diagnostics $\mathrm{GmbH}$, Mannheim, Germany). An open developmental channel was used for this application.

The determination of u-ORM was performed by using anti human rabbit Orosomucoid Immunoparticles (ref. no. OA504, Dako Denmark A/S, Glostrup, Denmark) and Reaction Buffer (ref. no. P01812, Dako Denmark A/S, Glostrup, Denmark). The antigen, antibody and buffer volumes were optimized during the validation period.

Considering that there are no commercially available urinary ORM calibrators and controls, dilution series of N Protein Standard SL for BN II Systems (ref. no. OQIM13, Siemens Healthcare Diagnostics $\mathrm{GmbH}$, Marburg, Germany) with $788 \mathrm{mg} / \mathrm{L}$ orosomucoid concentration were used for calibration and two different dilutions $(4.125$ and $0.825 \mathrm{mg} / \mathrm{L}$, respectively) of N/T Protein Control SL/L (ref. no. 
OQIN13, Siemens Healthcare Diagnostics GmbH, Marburg, Germany) were applied as controls. All the dilutions of calibrators, controls and samples were made by sterile $154 \mathrm{mmol} / \mathrm{L} \mathrm{NaCl}$ solution which was also used as blank sample.

The optimal assay volumes were assessed using a wide concentration range of the calibrator (0.1$31.5 \mathrm{mg} / \mathrm{L}$ ), and a working range below the equivalence zone was chosen (antibody excess). Twopoint end assay type was set using 10-minute reaction time. The wavelength used for the turbidimetric reaction was $546 \mathrm{~nm}$ and delta absorbance was calculated after pipetting the latex-antibody particles (self-blank, between 38-70 measuring points). The calibrator/control/sample volume was $7 \mu \mathrm{L}$ and the latex-antibody volume was $40 \mu \mathrm{L}$. Full calibration was performed by applying a six-point standard curve (blank plus five different dilutions of the calibrator) and the spline graph evaluation method was used. The assay was performed at $37^{\circ} \mathrm{C}$.

\section{Validation protocol}

The validation protocol of our u-ORM assay is based on the $2^{\text {nd }}$ edition of Eurachem guidelines (23). The analytical limits were obtained by using the absorbance data of 30 independent determinations of blank samples. Mean, standard deviation (SD) and coefficients of variation (CV) were calculated. Limit of blank (LOB) was determined as the mean absorbance value $+1.645 \times$ SD of the blank samples (24), limit of detection (LOD) was calculated as the blank mean $+3 \times \mathrm{SD}$, while limit of quantification (LOQ) as the blank mean value + $10 \times$ SD. The corresponding concentrations were defined using cubic spline interpolation of the absorbance data by the Origin Pro8 software (OriginLab Corporation, Northampton, USA). Functional sensitivity was evaluated from five independent dilution series of a urine sample $(0.025-0.390$ $\mathrm{mg} / \mathrm{L}$ ) measured on five consecutive days in two parallels. Functional sensitivity was set at the lowest $\mathrm{u}$-ORM concentration where the $\mathrm{CV}$ reached $20 \%$.

For intra- and inter-assay imprecision measurements we used dilutions of PreciControl ClinChem Multi 2 (PC2, ref. no. 05117216 190, Roche Diagnos- tics $\mathrm{GmbH}$, Mannheim, Germany) with $845 \mathrm{mg} / \mathrm{L}$ orosomucoid concentration. Four different dilution levels of PC2 were used for the assessment of intra-assay (within run) imprecision by ten parallel measurements on the same day and for that of inter-assay (between run) imprecision using duplicate measurements on ten consecutive days. Also, recovery was calculated from the measured and theoretical value at four levels of PC2 and was expressed as $\%$ ( $\mathrm{N}=40$ for each level). Linearity was determined by two parallel measurements of ten different dilutions of a urine sample in the range of 0.10-4.68 mg/L.

Five different urine samples (ranging from 0.52 to $4.11 \mathrm{mg} / \mathrm{L}$ ) were selected for stability studies. Specimens were divided into aliquots and were stored at $2-8{ }^{\circ} \mathrm{C}$ for 10 days. U-ORM was determined right after sample collection and on the $3^{\text {rd }}$, $5^{\text {th }}, 8^{\text {th }}$ and $10^{\text {th }}$ day, respectively. Urinary orosomucoid stability after freezing - thawing cycles was also assessed. Aliquots of the samples without any preservatives were frozen at $-70^{\circ} \mathrm{C}$ and subsequently thawed at $37^{\circ} \mathrm{C}$, then u-ORM was measured thereafter, five freezing - thawing cycles were performed with a simultaneous u-ORM determination.

\section{Method comparison}

A reference method for $u-O R M$ measurements is not available; therefore, our results measured by turbidimetric assay were compared to results obtained by western blotting which was previously used, too (21). Urine samples from healthy individuals and various patients were analyzed by western blotting using primary anti-orosomucoid antibodies (ref. no. Q0326, Dako Denmark A/S, Glostrup, Denmark) and secondary horseradish peroxidase conjugated anti-rabbit immunoglobulins (ref. no. P0217, Dako Denmark A/S, Glostrup, Denmark). For the chemiluminescent detection and immunoblotting evaluation a charged coupled device (CCD) camera and software (Syngene, Cambridge, UK) was used. Dilution series of the $\mathrm{N}$ Protein Standard SL (ref. no. OQIM13, Siemens Healthcare Diagnostics GmbH, Marburg, Germany) were used for calibration of the western blot method after densitometry by plotting the chemi- 
luminescence signal of the standards vs the standards' concentrations. A linear regression fit was applied $\left(R^{2}=0.996\right)$. For the quantitative evaluation of the samples in each blot the same urine sample with known u-ORM concentration of a healthy individual was applied as an internal standard.

\section{Subjects}

In order to determine the reference range for $\mathrm{u}$ ORM, healthy volunteers $(\mathrm{N}=72)$ were recruited between the age of 10 and 60 years among whom three age groups were created (10-20, 21-40, and 41-60 years, respectively). Healthy state was assessed if the person had no chronic illness, no complaints, and no symptoms on medical examination and did not take prescribed medicines. Accordingly, exclusion criteria were inflammation (based on hsCRP, white blood cell count and clinical signs), any kind of chronic diseases (based on medical records) or the lack of consent. U-ORM was also analyzed in patients with clinical diagnosis of Crohn's disease ( $\mathrm{N}$ $=28$ ) independently from disease activity and in patients with sepsis $(\mathrm{N}=30)$. Our study was performed from January 2014 to November 2015. The study was approved by the Regional Ethics Committee of the University of Pécs, Medical School in accordance with the Helsinki declaration (no. 4327.3162900/KK15/2011). Every patient and control individual was fully informed and written consent was obtained from all of them. Spontaneous random urine samples and venous blood were simultaneously obtained from the participants. After centrifugation (1500 x g, 10 minutes) of clotted blood and urine samples, supernatants were collected into aliquots and stored at $-70{ }^{\circ} \mathrm{C}$ until use. We measured urinary total protein (u-TP) and creatinine on Cobas 8000 analyzer (Roche Diagnostics $\mathrm{GmbH}$, Mannheim, Germany). We expressed our u-ORM data not only in $\mathrm{mg} / \mathrm{L}$ concentrations but also in u-ORM/ urinary creatinine (u-CREAT) ratio $(\mathrm{mg} / \mathrm{mmol})$ and in $u$ ORM/u-TP (\%) proportion. Also, hs-CRP and creatinine were measured from serum samples.

\section{Statistical analyses}

The distribution of our variables was determined by Shapiro-Wilk analyses. For comparison of the laboratory parameters of our patient groups Kruskal-Wallis test was used when the distribution was not normal. To assess gender differences the Mann Whitney $U$ test was performed. Reference interval for u-ORM, u-ORM/u-CREAT and u-ORM/uTP were determined at 95\% confidence interval. Passing- Bablok regression analyses and Bland-Altman plots were used to compare the methods. The continuous variables were expressed as medians with percentiles in case of non-normal distribution. A $p$ value of $P<0.05$ was considered as statistically significant. Statistical analyses were performed by MedCalc Statistical Software version 16.8 (MedCalc Software, Ostend, Belgium).

\section{Results}

\section{Validation data}

The calibration curve obtained from 22 independent calibrations is shown in Figure 1A. The calibrated working range for $\mathrm{u}$-ORM measurements was between $0.16-5.25 \mathrm{mg} / \mathrm{L}$. Samples at above 5.25 $\mathrm{mg} / \mathrm{L} \mathrm{u}$-ORM concentrations had to be diluted due to the hook effect experienced (Figure 1B) while those under the lowest calibration point $(0.16$ $\mathrm{mg} / \mathrm{L}$ ) of the assay could be determined down to at least of $0.08 \mathrm{mg} / \mathrm{L}$. LOB, LOD, LOQ of our U-ORM assay were calculated as previously described, and were $0.0095,0.02$ and $0.08 \mathrm{mg} / \mathrm{L}$, respectively. The functional sensitivity of our assay was assessed to be $0.03 \mathrm{mg} / \mathrm{L}$ (Figure 2A).

Both intra- and inter- assay imprecision was determined to be less than $5 \%$ of CV and even recovery of our u-ORM assay was found to vary between $95.69-102.51 \%$ (Table 1). The assay showed good linearity $\left(R^{2}=0.999\right)$ in the range of $0.1-4.68 \mathrm{mg} / \mathrm{L}$ (Figure 2B).

\section{Stability studies}

During 10 days of storage at $2-8{ }^{\circ} \mathrm{C}$ we found no significant difference in the recovery of u-ORM concentrations of the urine samples (97.2-109.2\%). Furthermore, we found no significant decrease in u-ORM concentrations after repeated freeze-thaw cycles. Even at the $5^{\text {th }}$ cycle the recovery remained quite acceptable as presented in Table 2. 
A

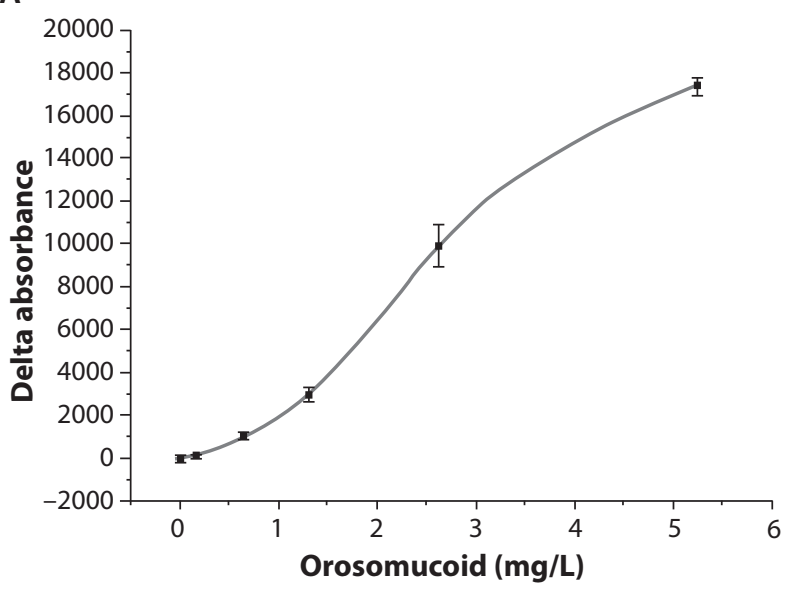

B

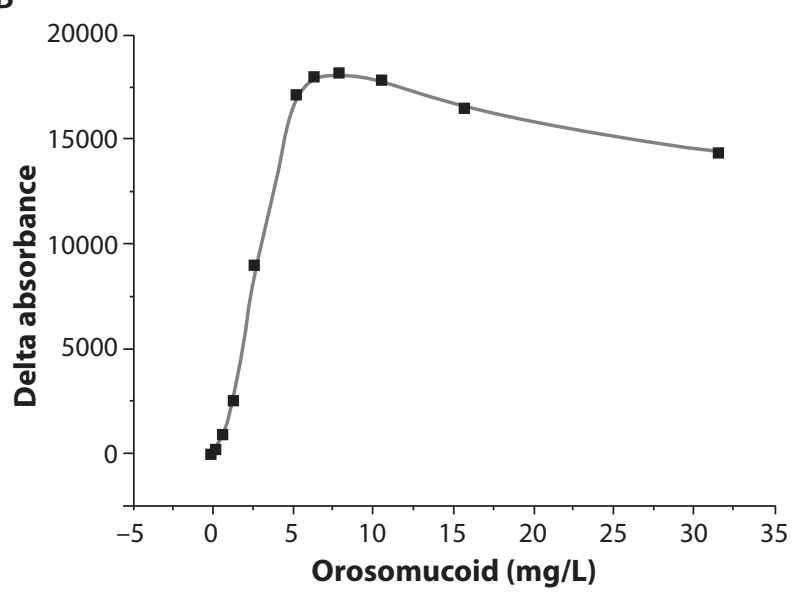

Figure 1. Calibration and dose response curve of u-ORM.

(A) Cumulative graph of a 6-point calibration curve of the assay in the range of $0.16-5.25 \mathrm{mg} / \mathrm{L}$ with spline graph fitting. Calibrators' mean \pm standard deviations from 22 separate calibrations are represented by red dots and bars.

(B) Dose response curve from serial dilutions of the calibrators in the range of $0.1-31.5 \mathrm{mg} / \mathrm{L}$.

A

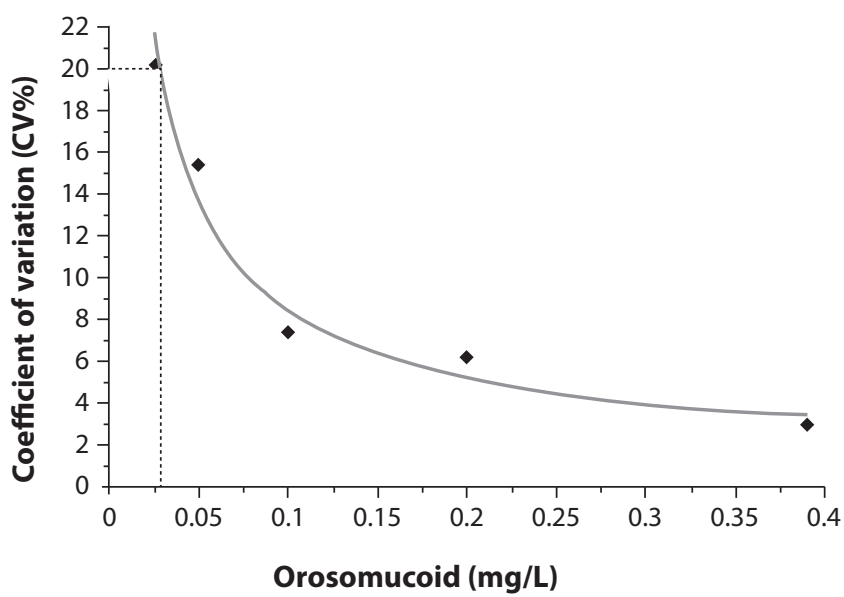

B

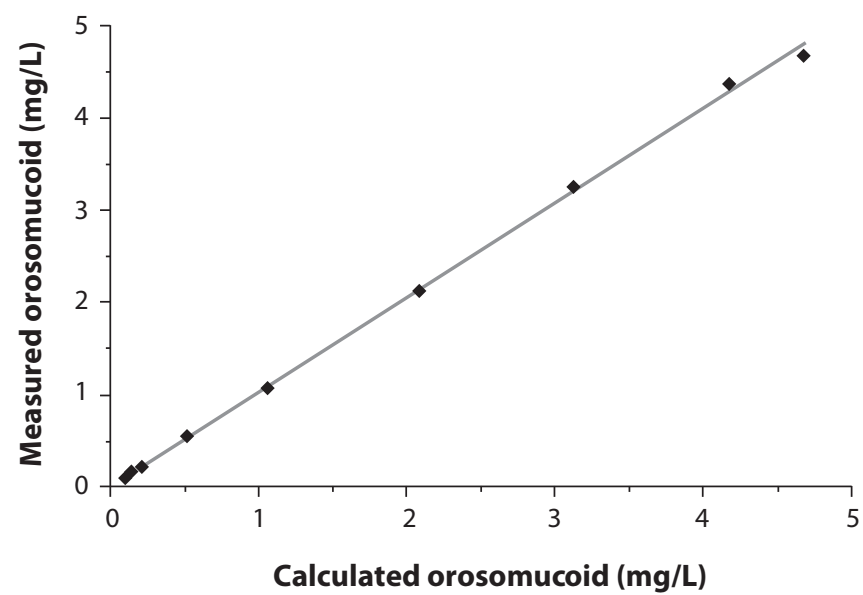

FIGURE 2. Functional sensitivity and linearity of the u-ORM assay. (A) Functional sensitivity was determined to be $0.03 \mathrm{mg} / \mathrm{L}$ (dashed line).

(B) Linearity was studied by diluting a urine sample within the range of $4.68-0.10 \mathrm{mg} / \mathrm{L}$ in 10 different dilutions. The regression line

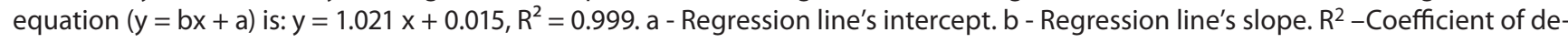
termination. Mean values of parallel measurements are presented.

TABLE 1. Imprecision and recovery data of u-ORM assay.

\begin{tabular}{cccccccc}
\hline \multirow{2}{*}{ Sample } & $\begin{array}{c}\text { Target } \\
\text { value, } \\
\mathbf{m g} / \mathbf{L}\end{array}$ & $\begin{array}{c}\text { Mean } \pm \mathrm{SD}, \\
\mathrm{mg} / \mathrm{L}\end{array}$ & $\mathrm{CV}, \%$ & Recovery, \% & Mean $\pm \mathrm{SD}, \mathrm{mg} / \mathrm{L}$ & $\mathrm{CV}, \%$ & Recovery, \% \\
\cline { 3 - 8 } & 3.86 & $3.73 \pm 0.08$ & 2.01 & 96.70 & $3.83 \pm 0.13$ & 3.50 & 99.24 \\
$\mathrm{C} 1^{*}$ & 2.11 & $2.06 \pm 0.01$ & 0.45 & 97.98 & $2.17 \pm 0.04$ & 1.77 & 102.51 \\
$\mathrm{C}{ }^{*}$ & 0.64 & $0.62 \pm 0.01$ & 1.92 & 95.69 & $0.63 \pm 0.03$ & 4.29 & 98.31 \\
$\mathrm{C3}^{*}$ & 0.30 & $0.30 \pm 0.01$ & 4.44 & 100.71 & $0.30 \pm 0.01$ & 4.55 & 100.18 \\
$\mathrm{C4}^{*}$ & & &
\end{tabular}

*Samples were dilutions from PC2 control. SD - standard deviation. CV - coefficient of variation. 
TABLE 2. Stability studies of urinary orosomucoid

\begin{tabular}{cccccccccc}
\hline \multirow{2}{*}{$\begin{array}{c}\text { Initial u-ORM } \\
\text { concentration, } \mathbf{~ m g / L}\end{array}$} & \multicolumn{4}{c}{ Storage at 2-8 ${ }^{\circ} \mathbf{C}$} & \multicolumn{4}{c}{ Thawing-freezing cycles } \\
\cline { 2 - 9 } & Day 3, \% & Day 5, \% & Day 8, \% & Day 10, \% & Cycle 1, \% & Cycle 2, \% & Cycle 3, \% & Cycle 4, \% & Cycle 5, \% \\
\hline 4.11 & 98.4 & 103.6 & 105.7 & 109.2 & 106.3 & 108.3 & 109.5 & 111.7 & 112.9 \\
3.61 & 99.1 & 101.5 & 102.4 & 106.6 & 105.5 & 106.7 & 106.4 & 105.3 & 104.6 \\
2.74 & 98.9 & 100.5 & 103.0 & 105.8 & 104.6 & 105.7 & 104.2 & 104.5 & 103.6 \\
1.23 & 97.2 & 98.8 & 99.1 & 107.9 & 106.3 & 108.2 & 109.4 & 112.6 & 113.1 \\
0.52 & 104.5 & 98.8 & $-*$ & $-*$ & 104.5 & 104.8 & 102.0 & 107.6 & 110.0 \\
\hline
\end{tabular}

Urinary ORM concentrations are referred to initial concentration and expressed in \%. *sample with $0.52 \mathrm{mg} / \mathrm{L}$ initial concentration could not be tested on day 8 and 10 because of non-expected sample error.

\section{Method comparison}

96 samples with a u-ORM concentration range of $0.23-292 \mathrm{mg} / \mathrm{L}$ were analyzed by western blotting and the new turbidimetric assay. We found a strong positive correlation $(r=0.924, p<0.001)$ between the results obtained by the two methods; however, the concentrations determined by western blotting were lower than those obtained by immune turbidimetry and a proportional difference between the methods was found. The comparison of the methods is illustrated in Figure 3.

\section{Reference range, patients}

The data of healthy individuals are listed in Table 3. We found no significant differences in U-ORM excretion between the three age-groups of healthy volunteers, therefore we suggest pooling all the data and using a common reference value for all cases between 10-60 years. Based on the spontaneous u-ORM concentrations of 72 healthy persons, the following reference intervals, expressed as median (2.5-97.5 percentiles), could be defined: $0.69(0.13-2.96) \mathrm{mg} / \mathrm{L}$ for u-ORM, $0.08(0.01-0.24)$
A

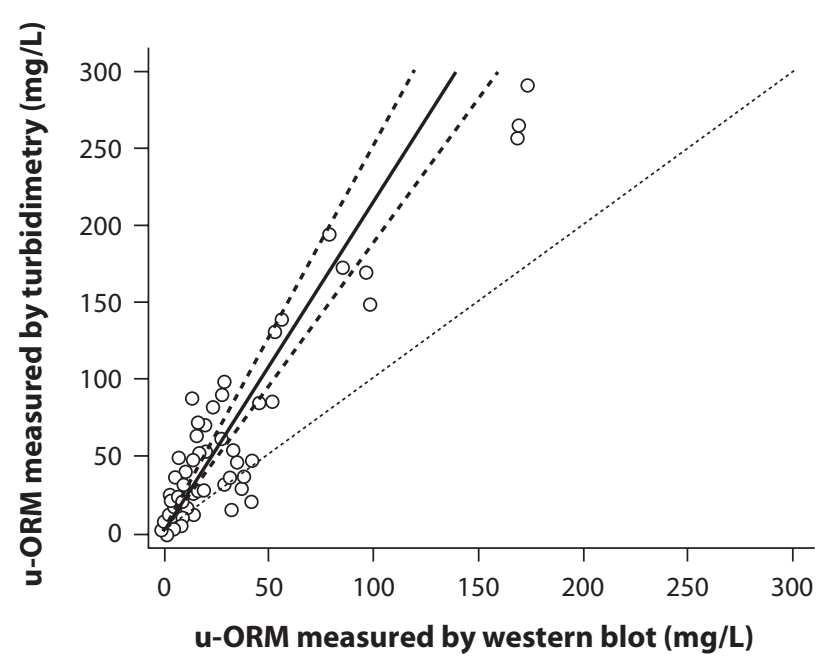

B

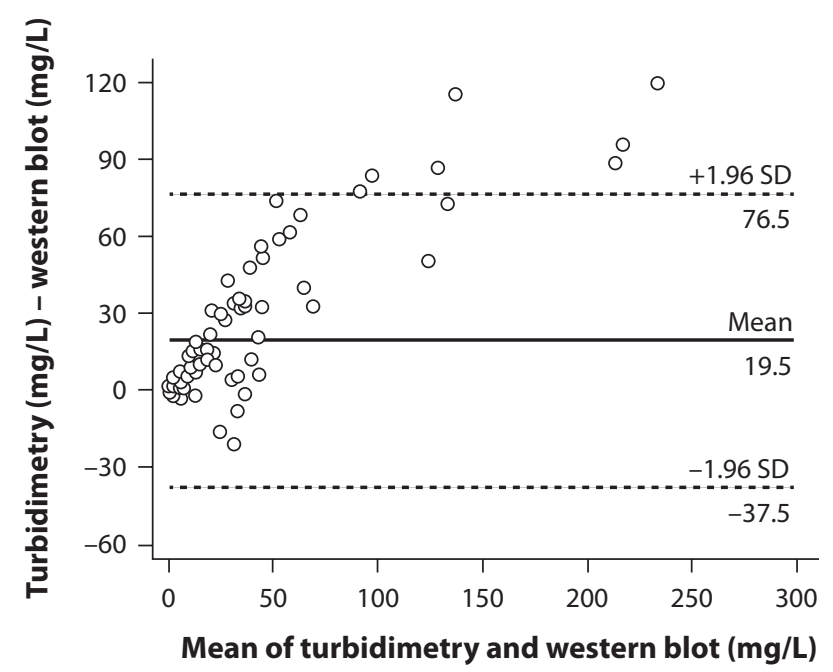

Figure 3. Comparison of quantitative western blotting and the new automated turbidimetric assay for u-ORM measurement.

(A) Passing-Bablok regression analysis. Regression line equation $(y=a(95 \% \mathrm{Cl})+\mathrm{b}(95 \% \mathrm{Cl}) \mathrm{x})$ is $\mathrm{y}=0.154(-0.396$ to 0.574$)+2.143$ (1.882 to 2.523) x. a - Regression line's intercept. b - Regression line's slope. $95 \% \mathrm{Cl}$ - 95\% confidence interval. Cusum test for linearity showed no significant deviation from linearity $(P=0.95)$. Solid line - regression line. Dashed lines $-95 \% \mathrm{Cl}$ for the regression line. Dotted line - identity line $(x=y)$.

(B) Bland-Altman plot of the mean concentration against the difference for the compared methods. Solid line (mean) - mean difference. Dashed lines (SD) - standard deviation. 
TABLE 3. Preliminary reference ranges of $u-O R M$ values in healthy individuals

\begin{tabular}{lccccc}
\hline & $\begin{array}{c}\text { Group 1, } \\
\mathbf{N = 3 0}\end{array}$ & $\begin{array}{c}\text { Group 2, } \\
\mathbf{N = 1 9}\end{array}$ & $\begin{array}{c}\text { Group 3, } \\
\mathbf{N = \mathbf { 2 3 }}\end{array}$ & $\begin{array}{c}\text { P } \\
\text { Pemales, N (proportion) }\end{array}$ & $\begin{array}{c}\text { Total control group, } \\
\mathbf{N = 7 2}\end{array}$ \\
\hline Age, years & $13(0.43)$ & $9(0.47)$ & $15(0.65)$ & NA & $37(0.51)$ \\
U-ORM, mg/L & $14(10-18)$ & $29(21-39)$ & $51(40-58)$ & NA & $23(10-58)$ \\
U-ORM / u-CREAT, mg/mmol & $0.56(0.10-2.96)$ & $0.71(0.25-2.30)$ & $0.76(0.14-2.64)$ & 0.730 & $0.69(0.13-2.96)$ \\
U-ORM / u-TP, \% & $0.09(0.02-0.19)$ & $0.07(0.01-0.35)$ & $0.08(0.02-0.24)$ & 0.590 & $0.08(0.01-0.24)$ \\
\hline
\end{tabular}

Data are presented as median and 2.5-97.5 percentiles. Age is presented as median (range). Kruskal-Wallis test was used to compare the different age groups of controls. $\mathrm{P}<0.05$ was considered statistically significant. NA-not applicable. U-ORM - urinary orosomucoid. u-CREAT - urinary creatinine. U-TP - urinary total protein.

$\mathrm{mg} / \mathrm{mmol}$ for $\mathrm{u}$-ORM/u-CREAT and 1.00 (0.213.58)\% for u-ORM/u-TP proportion. There was no significant difference in u-ORM/u-CREAT between females and males in the three control groups. However, significantly elevated u-ORM concentration $(P=0.034)$ and $u$-ORM/u-TP $(P=0.023)$ were observed in males compared to females $(1.3 \mathrm{mg} / \mathrm{L}$ and $2.29 \%, 0.49 \mathrm{mg} / \mathrm{L}$ and $0.62 \%$, respectively) in the age group of 41-60 years. Regarding past medical history and laboratory data (serum creatinine concentrations) in our control group, normal kidney function was hypothesized and also no proteinuria and no inflammatory activity were observed in healthy individuals.

We found significantly elevated $(P<0.001)$ u-ORM concentrations in inflammatory diseases (Table 4).
Compared to controls, about a 5-fold increase of u-ORM/u-CREAT ratios in Crohn's disease $(0.40$ $\mathrm{mg} / \mathrm{mmol}, \mathrm{P}<0.001)$ and an extreme, approximately 240 -fold elevation in sepsis $(19.13 \mathrm{mg} /$ mmol, $\mathrm{P}<0.001$ ) was found. The observed $\mathrm{u}$-ORM concentration in severe sepsis was significantly higher $(P<0.001)$ than that in morbus Crohn. Likewise, significant elevation was discovered in uORM/u-TP proportions both in sepsis and in Crohn's disease compared to healthy people $(\mathrm{P}<$ 0.001).

\section{Discussion}

In the present study we reported the adaptation and validation of an automated immune turbidi-

TABLE 4. Urinary orosomucoid concentrations in inflammatory diseases

\begin{tabular}{|c|c|c|c|c|}
\hline & $\begin{array}{l}\text { Total control group, } \\
\qquad N=72\end{array}$ & $\begin{array}{c}\text { Crohn's disease, } \\
\qquad \mathbf{N}=\mathbf{2 8}\end{array}$ & $\begin{array}{l}\text { Sepsis, } \\
N=30\end{array}$ & $\mathbf{P}$ \\
\hline Females, N (proportion) & $37(0.51)$ & $15(0.54)$ & $11(0.37)$ & NA \\
\hline Age, years & $23(10-58)$ & $27(12-62)$ & $67(30-82)$ & NA \\
\hline Serum creatinine, $(\mu \mathrm{mol} / \mathrm{L})$ & $64(55-75)$ & $59(50-71)$ & $130(104-181)$ & $<0.001$ \\
\hline hs-CRP, mg/L & $0.60(0.30-1.50)$ & $6.10(2.95-14.16)$ & 260.09 (158.16-335.73) & $<0.001$ \\
\hline U-ORM, mg/L & $0.69(0.33-1.13)$ & $4.15(1.97-10.30)$ & $90.21(42.01-137.23)$ & $<0.001$ \\
\hline U-ORM / u-CREAT, mg/mmol & $0.08(0.05-0.15)$ & $0.40(0.20-1.08)$ & $19.13(13.04-32.80)$ & $<0.001$ \\
\hline U-ORM/u-TP, \% & $1.00(0.54-1.69)$ & $3.72(2.48-6.41)$ & $18.71(11.88-34.86)$ & $<0.001$ \\
\hline
\end{tabular}

Data are presented as median and 25-75 percentiles. Age is presented as median (range). Kruskal-Wallis test was used to compare variables in different groups. $\mathrm{P}<0.05$ was considered statistically significant. NA - not applicable. Hs-CRP - high sensitive C-reactive protein. U-ORM - urinary orosomucoid. u-CREAT - urinary creatinine. U-TP - urinary total protein. 
metric assay for u-ORM measurements on Cobas $8000 /$ c502 platform, and also pointed out its potential clinical relevance.

This new turbidimetric approach was set up to be fast, sensitive and precise, therefore it is ideal for routine $\mathrm{u}$-ORM determinations. The imprecision of our method was tested by using diluted controls with a CV at around 5\%. Therefore, taken real urine samples we accepted the quality requirements for this assay with an imprecision of less than $10 \%$ as $\mathrm{CV}$. The test requires only 10 minutes. Due to the extraordinarily low LOQ $(0.08 \mathrm{mg} / \mathrm{L})$ and the good functional sensitivity $(0.03 \mathrm{mg} / \mathrm{L})$ our PET assay enables the quantitative $\mathrm{u}$-ORM determination in all healthy individuals and, after proper dilution in patients suffering from systemic inflammatory diseases. However, due to the narrow security zone, a 20 -fold predilution of all urine samples is suggested in order to automatically extend the measurable concentration limit up to $105 \mathrm{mg} / \mathrm{L}$. Samples containing low orosomucoid concentrations $(<1$ $\mathrm{mg} / \mathrm{L}$ ) can be retested without dilution however these samples' data has no clinical relevance.

Although, u-ORM seems to be a promising marker in diseases with systemic inflammatory activation, up to now only Christiansen et al. described an automated turbidimetric immunoassay for u-ORM determination similar to ours (25). Nevertheless, our assay settings allow more sensitive determination compared to their detection limit $(0.05 \mathrm{mg} / \mathrm{L})$; moreover our method seems to be more precise in the lower concentration range.

In agreement with other studies we also proved that ORM in urine is stable in vitro $(25,26)$. Our results showed that within 10 days the u-ORM concentrations are considerably constant in samples without additives and stored at $2-8{ }^{\circ} \mathrm{C}$. Furthermore, we found no loss in u-ORM concentration during 5 freezing-thawing cycles. These results support that urine samples can be stored at $2-8^{\circ} \mathrm{C}$ even for 10 days before analysis, and in case of delayed analysis for any reason, samples can be frozen and tested later.

In our preliminary studies, we found dramatically higher u-ORM concentrations in sepsis by immunoblotting $(21,22)$. Good correlation between the immunoblotting results and this PET immunoassay was found, although the methods are not directly comparable due to systemic bias. The use of quantitative u-ORM determination by immunoblotting, on the grounds of the methodological uncertainty, imprecision and time demand means that western blotting is unsuitable for daily routine u-ORM testing.

In our present work, we found a low urinary orosomucoid excretion rate in healthy persons. The expression of urinary protein concentrations referred to urinary creatinine is necessary in order to reduce the large inter-individual variability in the amount of excreted protein of urine samples. Based on spot urine samples of 72 healthy volunteers from different age groups between 10-60 years, we determined a preliminary reference range for $\mathrm{u}$-ORM/u-CREAT to be $0.01-0.24 \mathrm{mg} /$ $\mathrm{mmol}$ with a median of $0.08 \mathrm{mg} / \mathrm{mmol}$. Our results are in accordance with former studies $(25,27,28)$. Tencer et al. analyzed 95 healthy adults and established an upper reference limit for u-ORM/u-CREAT at $0.7 \mathrm{mg} / \mathrm{mmol}$. They found no correlation with age and found no differences between the gender and the type of urine collection (27). Hjorth et al. determined a cut off value for u-ORM/u-CREAT by single radial immunodiffusion of $0.5 \mathrm{mg} / \mathrm{mmol}$ in 233 children and in infants at the age from 1 month to 15 years. They found significantly higher u-ORM concentrations in neonates in the first month (28). Christiansen et al. described a similar reference range to ours for $\mathrm{u}$-ORM/u-CREAT of $0.009-0.17 \mathrm{mg} / \mathrm{mmol}$, with a median value of 0.04 $\mathrm{mg} / \mathrm{mmol}$ measured by their u-ORM PET assay. In the examined 69 adults with mean age of 43 years they also found higher urinary orosomucoid excretion in men than in women however, the differences disappeared when results were corrected to body surface area (25). We also noticed significant differences in u-ORM concentration between females and males above 40 but if $u$-ORM were corrected to $u$-CREAT, as expectable no differences were observed. Therefore, u-ORM level is suggested to be expressed as urinary orosomucoid/creatinine ratio in order to make results easier to interpret in different patients. We conclude that our preliminary u-ORM reference values seem to be 
suitable for both children and adults, furthermore our method is precise and sensitive enough to measure urinary orosomucoid in every healthy individual.

To date no studies have been reported regarding the u-ORM concentrations in Crohn's disease, however serum ORM is a well-known marker of morbus Crohn (29). Our observations suggest that elevated u-ORM may also provide additional information on this disease. So far, in sepsis only one paper with 7 septic patients reported a 280-fold increase of u-ORM excretion, which is similar to our results based on a larger sample size (10). The observed early and extreme elevation of u-ORM indicates that it could be a potential biomarker of sepsis.

During urinalysis we further observed a relative increase in u-ORM/u-TP proportion - both in Crohn's disease and in sepsis, which indicates that ORM becomes a prominent protein component in urine during inflammation. The observed increase of $u$ ORM values in our patient groups supports the hypothesis that u-ORM elevation is caused by inflammatory activity. Although the cause of such elevation is probably multifactorial, both systemic inflammation and renal factors are supposed to be responsible for it. Based on our results, we further support the hypothesis that u-ORM is a sensitive marker of systemic inflammation.

This study has several limitations. Although, we demonstrated examples for the possible clinical utilization of u-ORM measurements, this study reports only the analytical validation of u-ORM assay, and it is not a diagnostic accuracy study (30). Further research is required to describe the diagnostic performance of u-ORM in inflammatory diseases. Calibrators, control materials and a reference method are not commercially available for uORM measurements, only for serum testing. Therefore, in our u-ORM assay validation diluted

\section{References}

1. Schmid K. a1-acid glycoprotein. In: Putnam FW, ed. The plasma proteins. New York: Academic Press; 1975. p.183-228. http://dx.doi.org/10.1016/B978-0-12-568401-9.50011-6. serum calibrators/controls were used. For these reasons, the accuracy of our test could not be determined; rather recovery was calculated from the appropriate dilutions of PC2. The functional sensitivity was tested down to $0.025 \mathrm{mg} / \mathrm{L}$ ORM, where the sample's CV exceeded $20 \%$. The reported preliminary reference range was established by investigation of a relatively small healthy population. The robustness of our assay could not be estimated on other automated analyzers. Furthermore, we may refer only to potential interferences of the assay, which were tested previously, and no interference was found with albumin $(20 \mathrm{~g} / \mathrm{L})$, hemoglobin $(5 \mathrm{~g} / \mathrm{L})$, bilirubin $(600 \mathrm{mg} / \mathrm{L})$, creatinine $(5$ $\mathrm{g} / \mathrm{L}$ ) and glucose (100 $\mathrm{g} / \mathrm{L})(25)$.

In conclusion, we described the validation of a sensitive, precise and fully automated particle-enhanced immune turbidimetric assay for urinary orosomucoid which serves early and valuable information on inflammatory diseases. Our assay is ideal for routine clinical utilization to measure uORM as a possible novel marker of inflammatory activity.

\section{Acknowledgements}

The present scientific contribution is dedicated to the 650th anniversary of the foundation of the University of Pécs, Hungary. The work was financially supported by GINOP-2.3.2-15-2016-00021 "The use of chip-technology in increasing the effectiveness of human in vitro fertilization" and NKFI-EPR K/115394/2015 "Early biochemical indicators of embryo viability" grants. Hereby we express our special thanks to Roche Magyarország Kft. for their support, and also the invaluable technical help of Zoltán Horváth (Roche Magyarország Kft.) is highly appreciated.

\section{Potential conflict of interest}

None declared.

2. Fournier T, Medjoubi-N N, Porquet D. Alpha-1-acid glycoprotein. Biochim Biophys Acta 2000;1482:157-71. http:// dx.doi.org/10.1016/S0167-4838(00)00153-9. 
3. Israili ZH, Dayton PG. Human alpha-1-glycoprotein and its interactions with drugs. Drug Metab Rev 2001;33:161-235. http://dx.doi.org/10.1081/DMR-100104402.

4. Haraldsson BS, Johnsson EK, Rippe B. Glomerular permselectivity is dependent on adequate serum concentrations of orosomucoid. Kidney Int 1992;41:310-6. http://dx.doi. org/10.1038/ki.1992.43.

5. Sörensson J, Matejka GL, Ohlson M, Haraldsson B. Human endothelial cells produce orosomucoid, an important component of the capillary barrier. Am J Physiol 1999;276:H530-4.

6. Hochepied T, Berger FG, Baumann H, Libert C. a1-Acid glycoprotein: an acute phase protein with inflammatory and immunomodulating properties. Cytokine Growth Factor Rev 2003;14:25-34. http://dx.doi.org/10.1016/S13596101(02)00054-0.

7. Ceciliani F, Pocacqua V. The acute phase protein a1-acid glycoprotein: a model for altered glycosylation during diseases. Curr Protein Pept Sci 2007;8:91-108. http://dx.doi. org/10.2174/138920307779941497.

8. Christiansen MS, Hommel E, Friberg L, Molvig J, Magid E, Feldt-Rasmussen B. Increased urinary orosomucoid excretion rate is not related to impaired renal function in patients with type 2 diabetes. J Diabetes Complications 2010;24:2836. http://dx.doi.org/10.1016/j.jdiacomp.2008.08.001.

9. Poortmans JR, Haralambie G. Biochemical changes in a 100 km run: proteins in serum and urine. Eur J Appl Physiol Occup Physiol 1979;40:245-54. http://dx.doi.org/10.1007/ BF00421516.

10. Magid E, Guldager $H$, Hesse D, Christiansen MS. Monitoring urinary orosomucoid in acute inflammation: observations on urinary excretion of orosomucoid, albumin, alpha1microglobulin, and IgG. Clin Chem 2005;51:2052-8. http:// dx.doi.org/10.1373/clinchem.2005.055442.

11. Kronborg CS, Allen J, Vittinghus E, Knudsen UB. Pre-symptomatic increase in urine-orosomucoid excretion in pre-eclamptic women. Acta Obstet Gynecol Scand 2007;86:930-7. http://dx.doi.org/10.1080/00016340701371322.

12. Christiansen MS, Hesse D, Ekbom P, Hesse U, Damm P, Hommel $E$, et al. Increased urinary orosomucoid excretion predicts preeclampsia in pregnant women with pregestational type 1 diabetes. Diabetes Res Clin Pract 2010;89:16-21. http://dx.doi.org/10.1016/j.diabres.2010.03.018.

13. Suzuki M, Wiers K, Brooks EB, Greis KD, Haines K, Klein-Gitelman $M S$, et al. Initial validation of a novel protein biomarker panel for active pediatric lupus nephritis. Pediatr Res 2009;65:530-6. http://dx.doi.org/10.1203/PDR.0b013e31819e4305.

14. Irmak S, Tilki D, Heukeshoven J, Oliveira-Ferrer L, Friedrich $M$, Huland $H$, et al. Stage dependent increase of orosomucoid and zinc-alpha-2-glycoprotein in urinary bladder cancer. Proteomics 2005;5:4296-304. http://dx.doi.org/10.1002/ pmic.200402005.

15. Christiansen MS, Iversen $K$, Larsen CT, Goetze JP, Hommel $E$, Molvig J, et al. Increased urinary orosomucoid excretion: a proposed marker for inflammation and endothelial dysfunction in patients with type 2 diabetes. Scand J Clin Lab Invest 2009;69:272-81. http://dx.doi. org/10.1080/00365510802531100.

16. Jiang H, Guan G, Zhang R, Liu G, Liu H, Hou X, et al. Increased urinary excretion of orosomucoid is a risk predictor of diabetic nephropathy. Nephrology (Carlton) 2009;14:332-7. http://dx.doi.org/10.1111/j.1440-1797.2008.01053.x.
17. Christiansen MS, Hommel E, Magid E, Feldt-Rasmussen B. Orosomucoid in urine is a powerful predictor of cardiovascular mortality in normoalbuminuric patients with type 2 diabetes at five years of follow-up. Diabetologia 2005;48:38693. $\mathrm{http} / / / d x . d o i . o r g / 10.1007 / \mathrm{s} 00125-004-1630-1$.

18. Christiansen MS, Hommel E, Magid E, Feldt-Rasmussen B. Orosomucoid in urine predicts cardiovascular and overall mortality in patients with type II diabetes. Diabetologia 2002;45:115-20. http://dx.doi.org/10.1007/s125-002-8251-3.

19. Svendstrup M, Christiansen MS, Magid E, Hommel E, FeldtRasmussen $B$. Increased orosomucoid in urine is an independent predictor of cardiovascular and all-cause mortality in patients with type 2 diabetes at 10 years of followup. J Diabetes Complications 2013;27:570-5. http://dx.doi. org/10.1016/j.jdiacomp.2013.05.008.

20. Hou LN, Li F, Zeng QC, Su L, Chen PA, Xu ZH, et al. Excretion of urinary orosomucoid 1 protein is elevated in patients with chronic heart failure. PLoS One 2014;9:e107550. http:// dx.doi.org/10.1371/journal.pone.0107550.

21. Kustán P, Szirmay B, MühI D, Ludány A. Human orosomucoid in the clinical laboratory. In: Köszegi $T$, ed. Techniques with applicability in medical practice. Saarbrücken: LAP Lambert Academic Publishing; 2015. p.101-20

22. Kustan P, Ludany A, Muhl D, Horvath-Szalai Z, Koszegi T. Urinary orosomucoid in sepsis: laboratory approaches. Clin Chem Lab Med 2014;52:S1368.

23. Magnusson B, Örnemark $U$, eds. Eurachem Guide: The fitness for purpose of analytical methods - A Laboratory guide to method validation and related topics. 2nd ed. 2014. Available at: https://www.eurachem.org/index.php/publications/ guides/mv. Accessed June 23rd 2016.

24. Armbruster DA, Pry T. Limit of blank, limit of detection and limit of quantitation. Clin Biochem Rev 2008;29:S49-52.

25. Christiansen MS, Blirup-Jensen S, Foged L, Larsen M, Magid $E$. A particle-enhanced turbidimetric immunoassay for quantitative determination of orosomucoid in urine: development, validation and reference values. Clin Chem Lab Med 2004;42:1168-77. http://dx.doi.org/10.1515/CCLM.2004.237.

26. Tencer J, Thysell H, Andersson K, Grubb A. Stability of albumin, protein $H C$, immunoglobulin $G$, kappa- and lambda-chain immunoreactivity, orosomucoid and alpha 1-antitrypsin in urine stored at various conditions. Scand J Clin Lab Invest 1994;54:199-206. http://dx.doi. org/10.1080/00365519409088425.

27. Tencer J, Thysell H, Grubb A. Analysis of proteinuria: reference limits for urine excretion of albumin, protein HC, immunoglobulin G, kappa- and lambda-immunoreactivity, orosomucoid and alpha 1-antitrypsin. Scand J Clin Lab Invest 1996;56:691-700. http://dx.doi. org/10.3109/00365519609088816.

28. Hjorth L, Helin I, Grubb A. Age-related reference limits for urine levels of albumin, orosomucoid, immunoglobulin $G$ and protein HC in children. Scand J Clin Lab Invest 2000;60:6573. http://dx.doi.org/10.1080/00365510050185056.

29. Desai D, Faubion WA, Sandborn WJ. Review article: biological activity markers in inflammatory bowel disease. Aliment Pharmacol Ther 2007;25:247-55. http://dx.doi.org/10.1111/ j.1365-2036.2006.03184.x.

30. Šimundić AM. Measures of diagnostic accuracy: basic definitions. Available at: http://www.ifcc.org/ifccfiles/ docs/190404200805.pdf. Accessed September 16th 2016. 